\title{
Human Dimensions of Our Estuaries and Coasts
}

\author{
David Yoskowitz • Marc Russell
}

Received: 13 October 2014 /Revised: 4 December 2014 / Accepted: 5 December 2014 / Published online: 17 December 2014

(C) Coastal and Estuarine Research Federation 2014

\section{Introduction}

The connection between humans and the sea via the coastal margin is well understood. Many of our major cities are built in the coastal zone, and $44 \%$ of the world's population lives within $150 \mathrm{~km}$ of the coast (United Nations 2014a). This tight connection is driven by the benefits of commerce and the natural environment in the form of ecosystem goods and services. Our relationship with our coastal areas, however, is a delicate one. We receive numerous benefits but also have significant impacts on the systems providing these benefits. Quite simply, we often dominate our coasts, thus making the issue of governance even more relevant (Weinstein et al. 2007). Anthropogenic impacts, thus, eventually feedback and impact our well-being (MEA 2005; Cardinale, et al. 2012). Like many complex biogeochemical reactions, multiple ecosystem, economic, and social-cultural reagents combine in multiple ways to influence the end result. We propose here that the reagents of human well-being can be broken down to their elemental form.

In autumn of 2011, the Coastal and Estuarine Research Federation held its 21st biennial meeting. The theme of that conference was Societies, Estuaries, and Coasts: Adapting to Change. A unique aspect of this particular convening was the emphasis placed upon the interaction of humans with coastal environments, both as beneficiaries and sources of problems.

Communicated by Wayne S. Gardner

D. Yoskowitz $(\square)$

Harte Research Institute, Texas A\&M University-Corpus Christi, 6300 Ocean Drive, Corpus Christi, TX 78412, USA

e-mail: david.yoskowitz@tamucc.edu

M. Russell

Gulf Ecology Division, US EPA, One Sabine Island Drive, Gulf Breeze, FL 32561, USA
The substantial number of presentations focusing on human dimensions demonstrated an expertise not traditionally a part of biophysical scientist gatherings. The success of that assemblage spurred the idea for a special theme section of the Federation's journal, Estuaries and Coasts. This paper introduces that special section.

\section{A Foundation for Coastal Human Dimension Work}

This introductory article cannot capture the full breadth of coastal human dimension work, but we attempt to outline one way to consider the interaction between human society and our natural environment and policy options to help advance that understanding and put it into action.

Social-ecological models provide a framework to explore the idea of human-environment coupled systems, the basis of the articles for this special theme. As Dillard (2010) notes: "Attempts to work within a social-ecological model require concepts that extend between the two spheres, the social world and the ecological world, which have previously been separated by academic disciplines, research programs, and language." Where these distinct spheres do intersect is in the research and measurement of social-ecological resilience, the elucidation of how the resilience social systems are connected to the resilience of ecological systems (Adger 2000; Folke et al. 2002). The erosion of resilience is driven by not only environmental change but also human action that magnifies that change and can turn hazards into disasters (Adger et al. 2005). While social-ecological resilience studies may not be the end goal of many researchers, they contribute significantly to the "inter-" and "multidisciplinary" effort focused on the intersection of social and ecological systems (Dillard 2010).

The ability to conduct integrated studies is helped significantly by the increased demand or need for this type of work (policies) and therefore financial support, and the increased 
availability of approaches and tools that can help with the analysis and dissemination of results. However, the capacity to meet the perceived need in social-ecological work is still lacking, especially in social science disciplines (ISSC and UNESCO 2013); the "community" will need to address this need to effect real change. We present some of the approaches, tools, and policies that help bridge the gap between social and natural science disciplines as a prelude to the summary of the articles in this special theme section.

\section{Approaches and Tools}

Several approaches and corresponding tools are available to address the human dimensions of our coasts and the marine environment. A number of resources provide insight about the formal economy of the ocean and coasts (that which is captured in the marketplace). For example, the National Oceanic and Atmospheric Administration's (NOAA) ENOW Explorer (http://www.csc.noaa.gov/digitalcoast/tools/enow) includes a dataset, which captures the six most important economic sectors connected to the ocean and Great Lakes. The National Ocean Economics Program (http://www. oceaneconomics.org/) offers similar data on the ocean and coastal economy. But just as important are the intrinsic values that are not accounted for in the marketplace.

An important consequence of demonstrating the value of the environment not captured in the marketplace is providing a standardized protocol for assessment. The System for Environmental Accounting (SEEA) is an international standard for environmental-economic accounting developed by the UN (United Nations 2014b). It is designed to produce internationally comparable statistics and indicators to analyze trends across a broad spectrum of environmental and economic issues. The accounting framework described in SEEA Experimental Ecosystem Accounting uses both physical (i.e., liters of water filtered; hectares of marsh lost) and monetary terms. The use of physical indicators might be useful in circumstances where decision-makers and stakeholders are reluctant to put a monetary measurement on the environment but still want to capture its "value."

There are several efforts that incorporate human dimension into natural resource management at the national scale. The UK's National Ecosystem Assessment (UK National Ecosystem Assessment 2011) was introduced following the Millennium Ecosystem Assessment in response to the desire of the House of Commons to conduct a similar national assessment to recognize and minimize ecosystem service degradation. The desire was to

1. Assess the status and trends of the UK's ecosystems and the services they provide at multiple spatial scales.
2. Describe the key drivers of change affecting the UK's ecosystems, including changes in land use, infrastructure development, pollution, and climate.

3. Examine plausible futures (scenarios) for the UK's ecosystems and the services they provide.

4. Outline response options to secure continued delivery of the UK's ecosystem services for the benefit of all of society.

5. Value the contribution of ecosystem services to human well-being through economic and noneconomic analyses.

Several federal agencies are integrating human dimensions into research and policy activities in the USA. The US Environmental Protection Agency (US EPA) uses ecosystem-focused research to develop methods to measure ecosystem goods and services. The US EPA's Office of Research and Development (ORD) has developed several tools (i.e., EnviroAtlas, Final Ecosystem Goods and Services Classification System (FEGS-CS), Human Well-Being Index (HWBI), and National Ecosystem Services Classification System (NESCS)) to help resource managers consider human dimensions in their decision-making.

The EnviroAtlas is a collection of tools and resources that provides data, research, and analyses on the relationships between nature, people, health, and the economy (http:// enviroatlas.epa.gov/enviroatlas/atlas.html). The FEGS-CS (Landers and Nahlik 2013) defines and numbers each ecosystem benefit or service uniquely by combining environmental and beneficiary categories. This systematic approach is designed to minimize double counting in that it relates each FEGS to a defined beneficiary. It provides a standardized framework for linking ecosystems specifically to human well-being. The Human Well-Being Index (Smith et al. 2012) was developed as a core set of well-being domains that can be linked to ecosystem services via their relationship to economic, environmental, and societal well-being. The National Ecosystem Goods and Services Classification System (NEGSCS) extends the FEGS-CS by systematically mapping distinct pathways by which final ecosystem services enter and find value in human socioeconomic systems (http:// www.epa.gov/research/ecoscience/eco-negscs.htm). Accurate mapping through mutually exclusive categories on both the supply and demand side allows the separate calculation of one set of final ecosystem services against another-offering policy makers a tool to gauge how impacts to human wellbeing differ between one environmental management option and another.

The US Geological Survey (USGS) also recognizes the importance of representing the "social values" of ecosystem services in a spatially explicit manner. The development of a geographic information system (GIS) application, Social Values for Ecosystem Services (SolVES) (http://solves.cr. usgs.gov/), is designed to assess, map, and quantify the 
perceived social values of ecosystem services, such as aesthetics and recreation (Sherrouse et al. 2011). These nonmonetary values "...can be analyzed for various stakeholder groups as distinguished by their attitudes and preferences regarding public uses, such as motorized recreation or logging". SolVES is quantitative, producing a social value metric, the Value Index, from a blending of the spatial and nonspatial responses to case-specific surveys. Although originally applied to inland environments, SolVES is used in coastal ecosystem service research such as the recent work conducted by Florida Sea Grant in the Sarasota Bay area (https://www.flseagrant.org/waterways/ solves/).

An important component of these national efforts is classification of mappable units of ecosystem service production, especially in aquatic system where a lack of information exists. NOAA and its partners have developed the Coastal and Marine Ecological Classification Standard (CMECS) that provides a comprehensive national framework to organize information about coasts and oceans and their living systems. CMECS is designed for use within all waters ranging from the head of tide to the limits of the exclusive economic zone and from the spray zone to the deep ocean. A process to link CMECS habitat types to ecosystem services and help resource managers and researchers account for the connections between ecosystem structure, function, and services was described recently (Carollo et al. 2013).

Nongovernmental organizations (NGOs) also play critical roles in coastal human dimension work by connecting the work of academic and governmental institutions to decisionmakers and stakeholders. The Natural Capital Project (http:// www.naturalcapitalproject.org/) is a partnership between the World Wildlife Federation, The Nature Conservancy, Stanford University, and the University of Minnesota, with the goal to help governments and industry incorporate the value of nature's benefits into their development and conservation decisions. The World Resources Institute (WRI) has developed an Ecosystem Services Indicators Database “...to make ecosystem service metrics and indicators available for use in policy dialogs and decisions, in ecosystem assessments, and in natural resource management decisions" (http://www. esindicators.org/). The WRI also attempts to integrate environmental values into business decisions. Working with the World Business Council for Sustainable Development, they developed the Corporate Ecosystem Services Review (Hanson et al. 2012) with the goal to "...proactively develop strategies to manage business risks and opportunities arising from their company's dependence and impact on ecosystems. " The Marine Ecosystem Services Partnership (MESP), created as "... a virtual center for information and communication on the human uses of marine ecosystem services around the world," has developed a valuation database (http://www. marineecosystemservices.org/), which aggregates other existing databases such as the Gulf of Mexico Ecosystem Services Valuation Database (GecoServ), Ecosystem Services Valuation Database (ESVD), and ConsvalMap.org. The primary goal of MESP is to provide current and easily accessible data for any interested party from government agencies to academics and corporate managers.

\section{Policy}

In the USA, the ecosystem service approach is now considered in national and regional policy initiatives. The recent adoption of the new Principles and Requirements for Federal Investments in Water Resources (CEQ 2013) calls specifically for the inclusion of ecosystem services in the decision-making process. The US Department of Agriculture established the Office of Environmental Markets (formally Office of Ecosystem Services and Markets) with the directive to "... catalyze the development of markets for ecosystem services" given the unique position of the agency in the management of forests and rangelands (Forest Service 2014). Regional efforts such as the Gulf of Mexico Alliance Governors' Action Plan II calls for determining "...the socioeconomic value of critical coastal ecosystem services in the Gulf region" with the hope that these values will be considered in management decisions (GOMA 2009).

The National Oceanic and Atmospheric Administration's (NOAA) mission to sustain healthy coastal and marine habitat and the communities and economies that depend on them provides an opportunity for integrating human dimensions into coastal and marine resource management decisions. In 2007, the National Centers for Coastal and Ocean Science published the National Centers for Coastal Ocean Science Human Dimensions Strategic Plan (FY2009-FY2014) (NCCOS 2007), which established specific goals and objectives to integrate human dimensions into the science program to support coastal and ocean decision-making. Similarly, the National Marine Fisheries Service supports a human dimension program to support fisheries management decisions and includes "...fishing community profiles ... which contain social and economic data on fishing communities; the agency's social indicator effort for assessing community resiliency and vulnerability; Voices from the Fishery, an archive of oral histories from fishery participants, sociocultural studies, and science and management tools, data, and other resources."

In many cases, efforts by the United Nations and the European Union have accelerated the science and application of human dimensions of the environment and ecosystem services to planning and resource management decisions. The Economics of Ecosystems and Biodiversity (TEEB) is a global initiative, rooted in Europe, designed to attract attention to the socioeconomic benefits provided by ecosystems. The 
TEEB study follows a three-tiered approach in analyzing and structuring valuation:

1. Recognizing value in ecosystems, landscapes, species, and other aspects of biodiversity is a feature of all human societies and communities and is sometimes sufficient to ensure conservation and sustainable use.

2. Demonstrating value in economic terms is often useful for policy makers and business leaders in reaching decisions that consider the full costs and benefits of an ecosystem rather than just those costs or values that enter the markets in the form of private goods.

3. Capturing value involves the introduction of mechanisms that incorporate the values of ecosystems into decisionmaking through incentives and price signals.

\section{Social-Ecological Connections}

This special issue elucidates the inherent connections between human well-being and the natural environment. The socialecological cycle conceptual model we illustrate here includes the impact that humans have on natural resources and subsequent feedbacks to human well-being and how we respond to those changes through management dimensions (Fig. 1).

While the relationships between drivers and stressors and biophysical functions are often addressed by this journal, here, we emphasize the relationships between them and the other two components that complete the social-ecological cycle. The articles included in this special section on Human Dimensions of our Coasts fit within this framework. More specifically, these articles address the relationship between at

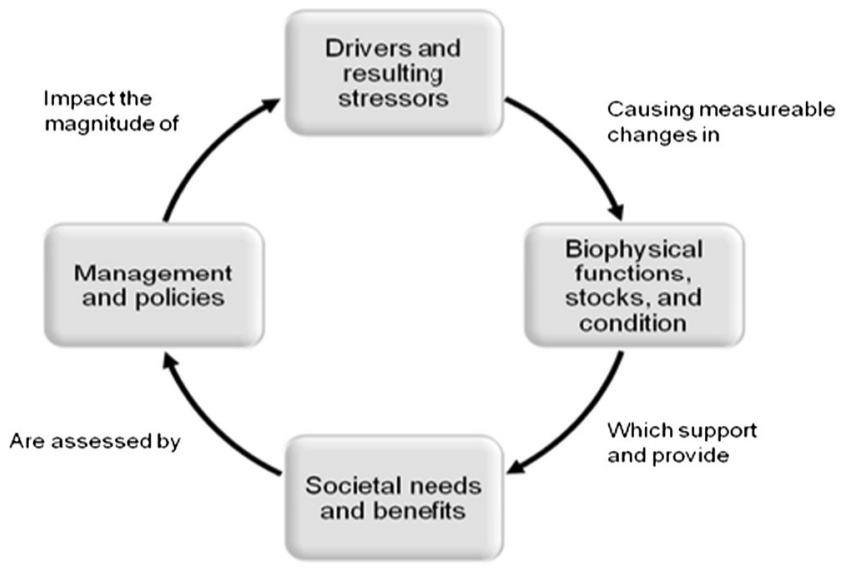

Components $=$ Relationships $=$

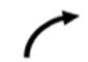

Fig. 1 Social-ecological cycle conceptual model illustrating the four components and major relationships explored in this paper least two components, with at least one being societal needs and benefits or management and policies. We recognize that more relationships occur among these four components within the social-ecological cycle than represented here, but this simplification helps compartmentalize the themes presented in this special theme issue. We consider the relationship between management and policies and drivers and stressors first to summarize how the authors in this special issue approached each relationship.

Relationships Between Management and Policies and Drivers and Stressors

Many management decisions and policies affect humans directly, while others cause directed shifts in forcing factors that result in an ecosystem response. While natural science papers often focus on the responses of ecosystems to changing drivers and stressors, we highlight several papers that explicitly mention this human dimension linkage in the socioecology cycle.

Restoration policies driven by observations of a degrading natural environment are designed to directly change the biophysical structure and function of ecosystems or increase human's ability to derive benefits from them. Russell and Greening (Estimating Benefits in a Recovering Estuary: Tampa Bay, Florida) estimated ecosystem goods and related potential cost savings for the Tampa Bay community from almost 20 years of seagrass expansion, coastal marsh and mangrove restoration/recovery, and habitats that have been maintained or preserved. This case study on the value of restoring a bay articulates the link between restoration policy and subsequent actions that have reduced excess nutrient inputs to the bay.

Wollheim et al. (Causes and Consequences of Ecosystem Service Regionalization in a Coastal Suburban Watershed) examine how communities in urbanizing watersheds respond to deficits in ecosystem service supply by moving across scales, such as by tapping into a neighboring watershed's water supply. The societal characteristics that lead to more sustainable solutions when mismatches between ecosystem service supply and demand occur were examined. The authors utilized a conceptual model to show how the provision of ecosystem services first expands, as rural population increases, and then contracts as management and policies move beyond the urbanized region. The model includes the human dimension of how communities manage supply and demand of ecosystem services to alleviate stress on the ecosystem by either finding remote replacements for those services or by conserving local supplies and limiting demand.

Needles et al. (Managing Bay and Estuarine Ecosystems for Multiple Services) demonstrate how managers are moving from a model of managing individual sectors, human activities, or ecosystem services to an ecosystem-based management (EBM) 
approach to balance the range of services provided by ecosystems. Applying EBM is often difficult because of inherent trade-offs in managing the different ecosystem services. This challenge is difficult for estuarine systems, which have been heavily altered in most regions and are often subjected to intense management interventions. A management service matrix is presented as a first step towards assessing trade-offs and providing managers with a decision-making tool. The management actions that restore or enhance natural vegetation (e.g., salt marsh and mangroves) and some shellfish (particularly oysters and oyster reef habitat) can enhance several ecosystem services. In contrast, management actions such as desalination, salt pond creation, sand mining, and large container shipping have net negative effects on ecosystem services.

Relationships Between Drivers and Stressors and Biophysical Functions, Stocks, and Condition

Agencies and researchers in the natural sciences are interested in how ecosystems respond to changes in stressors, often human-induced ones. Here, we highlight how this linkage in the socio-ecology cycle is approached by authors in this special section and how they integrate their work with the rest of the socio-ecology cycle.

Sanger et al. (Impacts of Coastal Development on the Ecology of Tidal Creek Ecosystems of the US Southeast Including Consequences to Humans) evaluate the impacts that coastal development, resulting land use changes, and nonpoint source pollution have on ecological condition of tidal creek ecosystems. They extended the biophysical condition endpoints using a range of exposure and response measures. The authors' illustrate how their research links to human dimensions within the socio-ecology cycle by updating a conceptual source-receptor model. The defined linkages are between coastal development and associated human activities, changes in the physical-chemical environment, anticipated responses of tidal creek ecosystems, and potential consequences to human populations and coastal communities.

Hutchison et al. (Stakeholder Perceptions of Coastal Habitat Ecosystem Services) link management of habitat to the provision of ecosystem goods and services by identifying and spatially quantifying the potential supply at the local level. This was a knowledge gap identified by the Millennium Ecosystem Assessment. While this is a secondary goal, behind their primary goal of exploring stakeholder perceptions of ecosystem goods and services, their mapping approach is the foundation, which their human dimension piece builds upon.

The expansion of the area supplying ecosystem services to humans is noted as a common response as coastal regions become more densely populated (Wollheim et al.; Causes and Consequences of Ecosystem Service Regionalization in a Coastal Suburban Watershed). This regionalization of supply areas has important consequences for watershed water supply and nitrogen $(\mathrm{N})$ fluxes to the coastal zone. The authors explored the link between biophysical changes in the Ipswich River watershed in northeastern Massachusetts, USA, and the water provisioning and nitrogen regulation. Historical information on water use and sources from the years 1850-2010 were utilized in the analysis.

Dellapenna et al. (Geological Responses to Urbanization of the Naples Bay Estuarine System, Southwestern Florida, USA) describe the extensive modifications caused directly and indirectly by anthropogenic influences in the NaplesDollar Bay Estuarine System (NDBES), situated in southwestern Florida, USA. These alterations include (1) substitution of mangrove-forested shorelines with concrete bulkheads and installation of residential canals; (2) installation of a regionally extensive navigational channel; and (3) canalization of the watershed, resulting in annexation of an altered drainage basin ten times the size of the pre-alteration basin and with significantly different soil and bedrock. The impact of anthropogenic alterations has shifted sediment distributions in northern Naples Bay significantly from a relatively coarser to a relatively fine-grained substrate. This transition has not occurred in southern Naples Bay and Dollar Bay because of the general lack of anthropogenic modifications to this part of the system.

Relationships Between Biophysical Functions, Stocks, and Condition and Societal Needs and Benefits

Extending typical biophysical response variables to changes in the benefits derived from them by humans is a key human dimension step in the socio-ecology cycle. Ecosystem attributes such as structure and function do not become ecosystem goods and services unless they are first valued, and then demanded, by human beneficiaries.

Paulisso et al. (Environmental Models and Public Stakeholders in the Chesapeake Bay Watershed) explored how new applications of the Chesapeake Bay Modeling System (CBMS) for regulatory efforts have increased public scrutiny of how models work. They describe a range of social, economic, political, and cultural issues that have emerged as a result of the CBMS being used to support mandatory nutrient reduction regulations. The articulation of local expert and scientific knowledge in terms that the public can understand and see value in is suggested as a critical need, with modelers, scientists, policy makers, and resource managers realizing the need to understand more of the human dimensions arising from the translation and implementation of the CBMS. They suggested a multidisciplinary approach is needed to integrate these rapidly emerging human societal and cultural dimensions into the expanded use of the CBMS.

A key component of the social-ecological cycle is the translation of biophysical variables into what human's value. Hutchison et al. (Stakeholder Perceptions of Coastal Habitat 
Ecosystem Services) utilized output from stakeholder workshops to assign weights to biophysical maps of the Coastal Bend region of Texas. The ecosystem variables were translated into metrics directly relatable to human beneficiaries. This augmented information serves as a guide during more informed resource management planning process.

Sanger et al. (Impacts of Coastal Development on the Ecology of Tidal Creek Ecosystems of the US Southeast Including Consequences to Humans) extended their impairment measurements in tidal creeks into a discussion of potential human health effects. They reaffirmed that tidal creeks, particularly the headwaters or intertidally dominated sections, are impaired by increases in nonpoint source pollution associated with sprawling urbanization. Links were established between human development decisions and behaviors and biophysical endpoints. They then completed the socio-ecology cycle by suggesting that tidal creek habitats may be valuable as early warning sentinels for identifying when ecological impacts from sprawling coastal development become potential public health and flooding risks.

Russell and Greening (Estimating Benefits in a Recovering Estuary: Tampa Bay, Florida) used several valuation techniques to translate ecosystem habitat extent measurements and stated restoration goals from biophysical terms to human benefits. They estimated that habitats in and around Tampa Bay provide nutrient reductions equivalent to just over US $\$ 22$ million per year in avoided wastewater treatment plant costs based on habitat that has been conserved or restored. They estimated an additional value accrual close to a quarter million dollars per year based on avoided societal costs from greenhouse gases that are sequestered by bay habitats and discuss how human beneficiaries include both the local and global community. These valuation estimates are useful for local resource managers pursuing continued funding for restoration efforts and help illustrate the public benefits of efforts that are often funded with public tax dollars.

The Wollheim et al. (Causes and Consequences of Ecosystem Service Regionalization in a Coastal Suburban Watershed) research suggests that specific human benefits derived from ecosystems, such as having adequate water supplies, change nonlinearly as societal demand for related ecosystem services increases in proportion to local and regional human activity. Their results illustrate how demand in densely populated areas can outstrip the ability of local systems to provide ecosystem services. Time series of ecosystem service sources, partitioned into locally or distally supplied or used at watershed scales, can help quantify how society responds to mismatches between ecosystem service demand and available sources by moving across scale. Sustainable local management is proposed as an alternative decision option but may require difficult choices that involve constraining human actions.
Savolainen et al. (Resiliency of the Coastal Recreational For-Hire Fishing Industry to Natural Disasters) examined the financial condition of US Gulf of Mexico recreation-for-hire (RFH) fishing firms. Post-hurricane damage was examined within the context of the industry's contribution to the resiliency of coastal social-ecological systems. Three key financial ratios - return-on-assets, assets turnover ratio, and debtto-assets ratio-were calculated for 2009 from balance sheets and cash flow statements constructed from surveys of 247 RFH firms operating in the five Gulf states. The ratios were then recalculated using reported damage and operational losses from at least one named storm in the 2004-2008 period and combined with the results of a logistic regression model of profitability loss to assess the resiliency of the RFH industry. Results suggest that RFH firm resiliency was a function of operating class (e.g., head, charter, and guide boats), home port, and the way in which the business was structured. Firms appeared to be the most resilient when they employed smaller vessels in intensively managed operations, perhaps because of their ability to move a vessel out of the path of storms and because their profitability and efficiency advantages allowed for self-insurance against losses. As a result, community contributions to, and benefits from, resiliency in the RFH industry may hinge on the development of more modern port facilities and well-functioning insurance markets.

Boucek and Rehage (A Tale of Two Fishes: Using Recreational Angler Records to Examine the Link Between Fish Catches and Floodplain Connections in a Subtropical Coastal River) suggested that, in the tropical and subtropical wet and dry regions, maintaining natural hydrologic connections between coastal rivers and adjacent ephemeral wetlands is critical to conserving and sustaining high levels of fisheries production within these systems. Although a consensus indicated that maintaining these natural connections is needed, little is known about what attributes of floodplain inundation regimes are most important in sustaining fisheries production. Using two Everglades coastal river fisheries as a model, they tested whether the amplitude of the flood season or the duration of the flood season is more important in explaining variation in angler catch records of common snook and largemouth bass collected from 1992 to 2012. They validated angler catches with fisheries-independent electrofishing conducted in the same region from 2004 to 2012.

\section{Relationships Between Societal Needs and Benefits and Management and Policies}

An assessment of the balance between the benefits humans derive from ecosystems and what they need or demand is a required prerequisite for setting new policy and managing our natural resources effectively. Human values, their perception of current conditions, and resulting behavior are influenced by and impact the local to global biophysical environment. 
Framing the biophysical condition in terms of valued ecosystem variables by using the concept of ecosystem goods and services and related benefits is the human dimension bridge within the socio-ecology cycle that allows biophysical measurements, models, and monitoring to effectively enter into planning discussions and the policy-making process.

Matso et al. (Funding Science that Links to Decisions: Case Studies Involving Coastal Land Use Planning Projects) noted that while the US Federal Government invests over a hundred billion dollars annually on science ${ }^{1}$, not enough science is linked with decisions. They report on three case studies involving new tools and science to help land use planners better protect coastal resources. Within the context of these representative case studies, they illustrated how increased efforts by funders to bring scientists and users into greater alignment can improve the chances that the resulting science is informative to decision-makers. Results from the three case studies also suggest that societal problems may be better addressed if funders do more to nudge science towards being more than the generation of new knowledge. They also suggest that funders need to focus significantly more attention and resources on the user engagement aspect of science projects. Their work is a good illustration that strong user engagement methods are an important human dimension in the socioecology cycle, on par with strong biophysical science or social science methods.

Hutchison et al. (Stakeholder Perceptions of Coastal Habitat Ecosystem Services) worked directly with stakeholders to identify perceptions of the value of ecosystem services provided by habitats in the Nueces Estuary. Their study demonstrates how a simple methodology for mapping the number of ecosystem services provided by habitats in the form of a heat map helped show stakeholders how they might benefit, at least in a relative sense, from alternatives in their natural resource management. Results of the study were incorporated into an ecosystem-based management plan. Their approach enabled stakeholders to make betterinformed decisions regarding priority areas for conservation, preservation, and restoration (http://www.cbbep.org/ projects/ecomanagement.html).

Ache et al. ("The Coast" is Complicated: A Model to Consistently Describe the Nation's Coastal Population) described how the nation's coast is a complicated management area where no single delineation provides all of the demographic statistics needed to address the full range of policy and management issues. As a result, several different coastal delineations are currently used, yielding a variety of US coastal population statistics. The paper proposes a simple model for generating and applying coastal population statistics at the

\footnotetext{
${ }^{1}$ The federal obligation for research in environmental science and biology in 2012 was \$4.6 billion (NSF, 2014).
}

national and regional level to increase consistency in coastal policy discussions and improve public understanding of coastal issues. The model includes two major components. The first component is "the population that most directly affects the coast," represented by the permanent US population that resides in a standard suite of coastal watershed counties, where land use and water quality changes most directly impact coastal ecosystems. The second component is "the population most directly affected by the coast," represented by the permanent US population that resides in a standard suite of coastal shoreline counties that are adjacent to the open ocean, major estuaries, and the Great Lakes, which bear a great proportion of the full range of effects from coastal hazards and host the majority of economic production associated with coastal and ocean resources.

\section{Conclusion}

A social-ecological approach, as described for this special issue, helps to connect these two traditionally disparate spheres. Human well-being is linked to the well-being of our natural environment and the effect of our actions on the natural environment. If these effects are negative, these lead to a potential reduction in community resilience.

The concept of humans receiving benefits from nature is anthropocentric by definition. Humans are part of the equation and need to be included when biophysical scientists generate models to estimate the production and flow of ecosystem function which results in the potential provision of ecosystem services. Ecosystem services result from the linkage of human needs and ecosystem function. But conservation and restoration decisions should not be based solely on ecosystem services. Like many complex biogeochemical reactions, multiple ecosystem, economic, and social-cultural reagents combine in multiple ways to influence the end result, but we propose here that the reagents of human well-being can be broken down to their elemental forms. Final ecosystem services are, at their core, one third of the elemental variables we need to account for when solving the human well-being equation. More traditional societal and economic variables constitute the other two thirds of those elements. Which variables limit the production of positive human well-being depends on basic stoichiometry of supply and demand which is spatially and temporally dynamic. Like an enzymatically catalyzed reaction, different types of communities and their well-being will respond to similar sets of reagents in different ways, with this response often modified by other influential factors from the surrounding setting. This observation is especially true during periods of scarcity, such as drought, or after ephemeral events such as hurricanes. The concept of ecosystem services transcends the boundaries of so-called natural and social science and 
provides the mechanism and language to merge those disciplines into a cohesive field of research.

Acknowledgments The authors appreciate the input from the Estuaries and Coasts co-editors-in-chief Iris Anderson, Wayne Gardner, and Charles "Si" Simenstad. We are especially grateful to Holly Greening for hatching this idea and providing critical support throughout the process. Views, opinions, and/or findings contained in this article are those of the authors and should not be construed as an official US Environmental Protection Agency position or decision.

\section{References}

Adger, W.N. 2000. Social and ecological resilience: Are they related? Progress in Human Geography 24: 347-364.

Adger, W.N., T.P. Hughes, C. Folke, S.R. Carpenter, and Johan Rockstrom. 2005. Social-ecological resilience to coastal disasters. Science 309: 1036-1039.

Cardinale, B.J., J.E. Duffy, A. Gonzalez, D.U. Hooper, C. Perrings, P. Venail, A. Narwani, G.M. Mace, D. Tilman, D.A. Wardle, A.P. Kinzig, G.C. Daily, M. Loreau, J.B. Grace, A. Larigauderie, D.S. Srivastava, and S. Naeem. 2012. Biodiversity loss and its impact on humanity. Nature 486(7): 59-67.

Carollo, C., R. Allee, and D.W. Yoskowitz. 2013. Linking the Coastal and Marine Ecological Classification Standard (CMECS) to ecosystem services: An application to the U.S. Gulf of Mexico. International Journal of Biodiversity Science, Ecosystem Services \& Management 9(3): 249-256. doi:10.1080/21513732.2013.811701.

CEQ. 2013. Principles and requirements for federal investments in water resources. March. http://www.whitehouse.gov/sites/default/files/ final_principles_and_requirements_march_2013.pdf

Dillard, M. 2010. Toward a measure of social-ecological resilience for human communities. In: Shifting shorelines: Adapting to the future, The 22nd International Conference of The Coastal Society, June 13-16, 2010, Wilmington, North Carolina. http://aquaticcommons.org/3898/

Folke, C., S. Carpenter, T. Elmqvist, L. Gunderson, C.S. Holling, and B. Walker. 2002. Resilience and sustainable development: building adaptive capacity in a world of transformations. AMBIO: A Journal of the Human Environment 31(5): 437-440.

Forest service. Retrieved February 11, 2014. http://www.fs.fed.us/ ecosystemservices/OEM/index.shtml
GOMA (Gulf of Mexico Alliance). 2009. Governors' Action Plan II. http://www.gulfofmexicoalliance.org/pdfs/ap2_final2.pdf\#view= Fit\&toolbar $=1$

Hanson, C., J. Ranganathan, C. Iceland, and J. Finisdore. 2012. The corporate ecosystem services review: Guidelines for identifying business risks and opportunities arising from ecosystem change. Version 2.0. Washington: World Resources Institute.

ISSC and UNESCO. 2013. World social science report 2013: Changing global environments. Paris: OECD Publishing and UNESCO Publishing.

Landers, D.H., and A.M. Nahlik. 2013. Final Ecosystem Goods and Services Classification System (FEGS-CS). EPA/600/R-13/ORD004914. Washington, DC: U.S. Environmental Protection Agency, Office of Research and Development.

Millenium Ecosystem Assessment (MEA). 2005. Ecosystems and human well-being, vol. 5. Washington: Island Press.

National Centers for Coastal Ocean Science. 2007. National Centers for Coastal Ocean Science Human Dimensions Strategic Plan (FY2009-FY2014). Silver Spring, MD: National Oceanic and Atmospheric Administration, National Ocean Service, National Centers for Coastal Ocean Science. 46 pp.

National Science Foundation (NSF). 2014. Federal funds for research and development. http://www.nsf.gov/statistics/nsf14316/content.cfm? pub id $=4418 \& \mathrm{id}=2$

Sherrouse, B.C., D.J. Semmens, and J.M. Clement. 2011. A GIS application for assessing, mapping, and quantifying the social values of ecosystem services. Applied Geography 31(2): 748-760.

Smith, L.M., H.M. Smith, J.L. Case, and L. Harwell. 2012. Indicators and methods for constructing a U.S. Human WellBeing Index (HWBI) for ecosystem services research. Washington, DC: U.S. Environmental Protection Agency. EPA/ 600/R-12/023, 2012.

UK National Ecosystem Assessment. 2011. The UK national ecosystem assessment technical report. Cambridge: UNEP-WCMC.

United Nations. 2014a. UN atlas of the oceans. Retrieved February 22, 2014. http://www.oceansatlas.org/servlet/CDSServlet?status= ND0xODc3JjY9ZW4mMzM9KiYzNz1rb3M

United Nations. 2014b. System of environmental accounting. Retrieved February 11, 2014. http://unstats.un.org/unsd/envaccounting/seea. asp

Weinstein, M.P., R.C. Baird, D.O. Conover, M. Gross, J. Keulartz, D.K. Loomis, Z. Naveh, S.B. Peterson, D.J. Reed, E. Roe, R.L. Swanson, J.A.A. Swart, J.M. Teal, R.E. Turner, and H.J. Van der Windt. 2007. Managing coastal resources in the 21st century. Frontiers in Ecology and the Environment 5: 43-48. doi:10.1111/j.1365-2664.2007. 01355.x. 